\title{
Mathematik in der zeitgenössischen bildenden Kunst
}

Robert Wolak

Die italienische Rennaissance des 15. Jahrhunderts - eine der glorreichsten Perioden der europäischen Kultur war eine Zeit großer Umwälzungen, in der Künstler Mathematik studierten und praktizierten und Mathematiker großes Interesse an Kunst zeigten. Gemeinsam entwickelten sie Methoden, um die Wirklichkeit zu erobern und legten die Grundlagen für die rasante Entwicklung der europäischen Kultur in den darauffolgenden Jahrhunderten.

Ich bin seit über 30 Jahren an Kunst interessiert. Die häufigste Reaktion, wenn ich preisgebe, dass ich Mathematiker bin, ist: „Ich hatte eine Menge Probleme mit Mathe in der Schule. Es war ein Wunder, dass ich die Abschlussprüfung bestanden habe ... " Meine Frau, die Mathematik an der Cracow Secondary Art School lehrt, hört sehr oft: „Warum sollten wir uns mit Mathematik beschäftigen? Wir sind Künstler, Geisteswissenschaftler ... “

Kunst und Mathematik haben jedoch viele Gemeinsamkeiten. Die Mathematik ist die Sprache, die das Universum wissenschaftlich beschreibt. Die Naturgesetze werden in mathematische Formeln gefasst. Und wahre Kunst, die ja nicht auf das Vergängliche gerichtet ist, ist bestrebt, die Welt und ihre Gesetze zu durchdringen und zu beschreiben. Kunst und Mathematik verfolgen also ähnliche Ziele, nur die Ansätze und Methoden sind verschieden.

Für den 6. Europäischen Mathematikerkongress (ECM), der im Juli 2012 in Krakau stattfand, beschloss ich, die Beziehungen zwischen Kunst und Mathematik in der Gegenwartskunst Krakaus, dem vielleicht wichtigsten kulturellen Zentrum Polens, zu beleuchten. Meine Recherche führte mich zu 14 Künstlern, von denen nur einer nicht in Krakau lebt und arbeitet. Das Ergebnis war eine Ausstellung zum 6. ECM mit dem Titel By Form and Number, die sehr gut besucht wurde. Im Folgenden zeigen wir eine (neu ergänzte) Auswahl der dort ausgestellten Werke.

Die Arbeiten zeigen, dass sich Künstler auf verschiedene Art und Weise mit Mathematik beschäftigen. Mathematische Objekte und Notationen werden zur Grundlage visueller Formen, geometrische Objekte sind nur eine von vielen Möglichkeiten. Natürlich sind geometrische Konstruktionen, Anamorphosen und Algorithmen beliebt. Manche Künstler suchen, wenn sie ein Objekt, ein Stück Natur, darstellen, nach einer inneren Struktur, nach einer Bedeutung, einem allgemein gültigen Prinzip. Diese Maler oder Bildhauer sind in vielerlei Hinsicht wie Mathematiker oder Wissenschaftler: Sie alle versuchen, die Rätsel der Welt zu lösen, aber sie erreichen ihr Ziel auf unterschiedlichen Wegen.

Der Titel der oben genannten Ausstellung ist übrigens von Platos Dialog „Timaeus“ abgeleitet. Lassen Sie mich in diesem Zusammenhang daran erinnern, dass über dem Eingang zu Platos Akademie folgender Satz geschrieben stand: ,Lasst niemanden eintreten, der nicht geometrisch denken kann." Ich frage mich, wie vielen Geisteswissenschaftlern heute wohl Einlass gewährt würde?

Der Katalog der Ausstellung wurde 2014 vom Polskie Towarzystwo Wydawcow Ksiazek (Polnischer Verband der Buchverlage) als das schönste in Polen publizierte Kunstbuch ausgezeichnet:

Robert Wolak, Formy i liczby. Copernicus Center Press, Krakow 20I4. ISBN 978-83-7886-I0I-0.

Dr. Robert Wolak, Instytut Matematyki, Uniwersytetu Jagiellońskiego, ul. Gołębia 24, 31-007 Kraków, Polen robert.wolak@im.uj.edu.pl

Robert Wolak wurde 1955 in Krakau geboren und ist dort aufgewachsen. Heute arbeitet er an der Fakultät für Mathematik und Computerwissenschaft der Jagellonischen Universität. Er wurde 1982 promoviert und habilitierte sich 1992. Seine Forschungsgebiete sind Differentialgeometrie und Topologie, Theorie der Blätterungen. Er war Mitglied des Executive Organizing Committee des 6. ECM, Kurator verschiedener Ausstellungen moderner polnischer Kunst und ist Sammler zeitgenössischer Kunst.

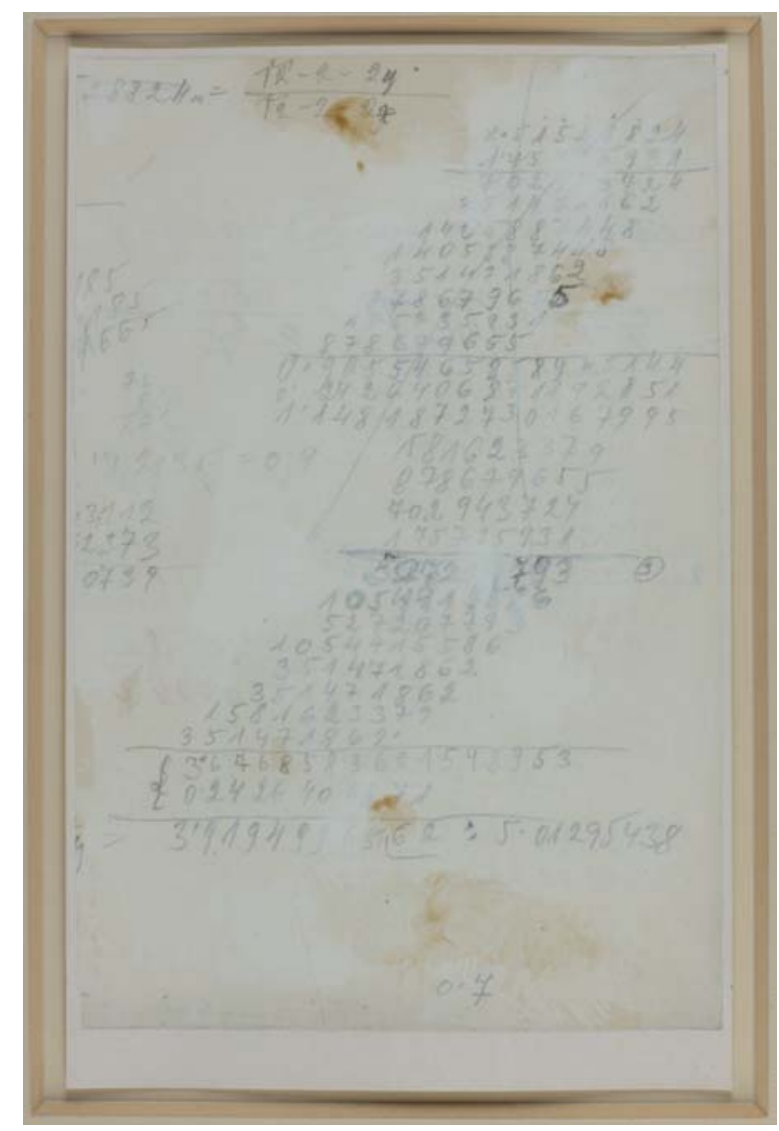

Marian Warzecha, Ohne Titel, mixed media, Papier, $21 \times 13,8 \mathrm{~cm}$, 2000/2002. 


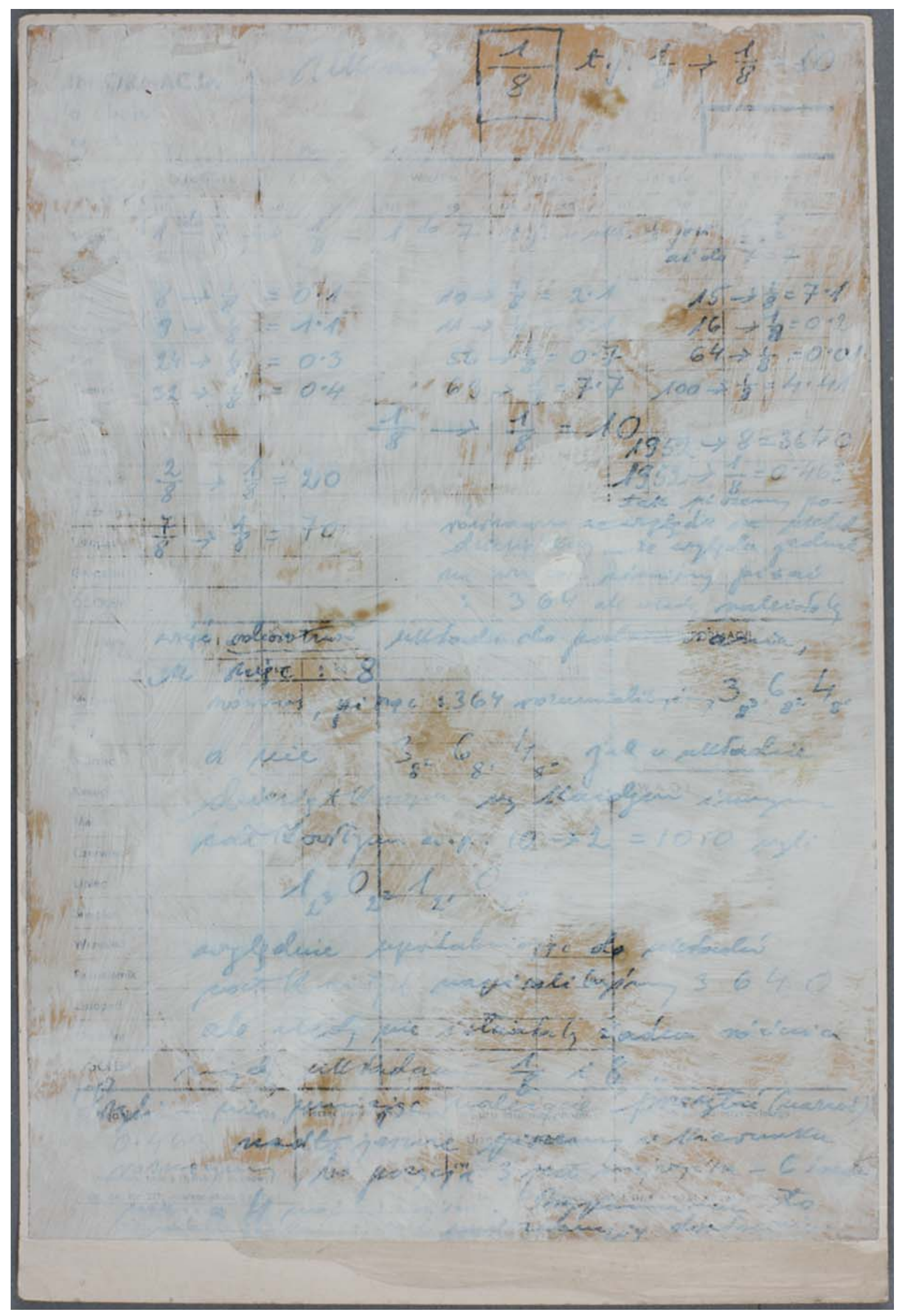

Marian Warzecha, Ohne Titel, mixed media, Papier, $30 \times 20,8 \mathrm{~cm}, 2000 / 2002$. 


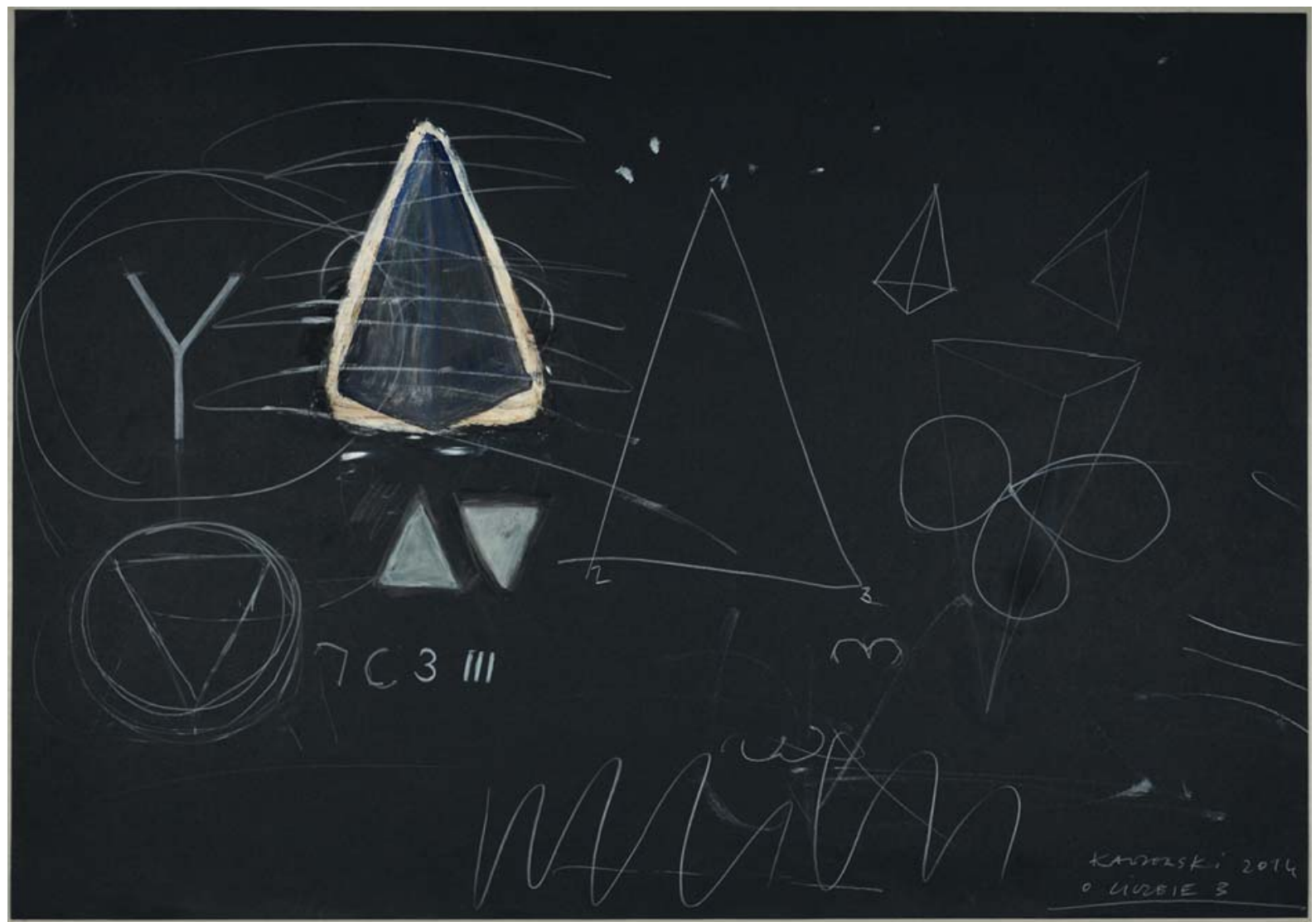

Jarosław Kawiorski, Über die Zahl 3, $70 \times 100 \mathrm{~cm}, 2014$, Wasserfarbe, Kreidestift

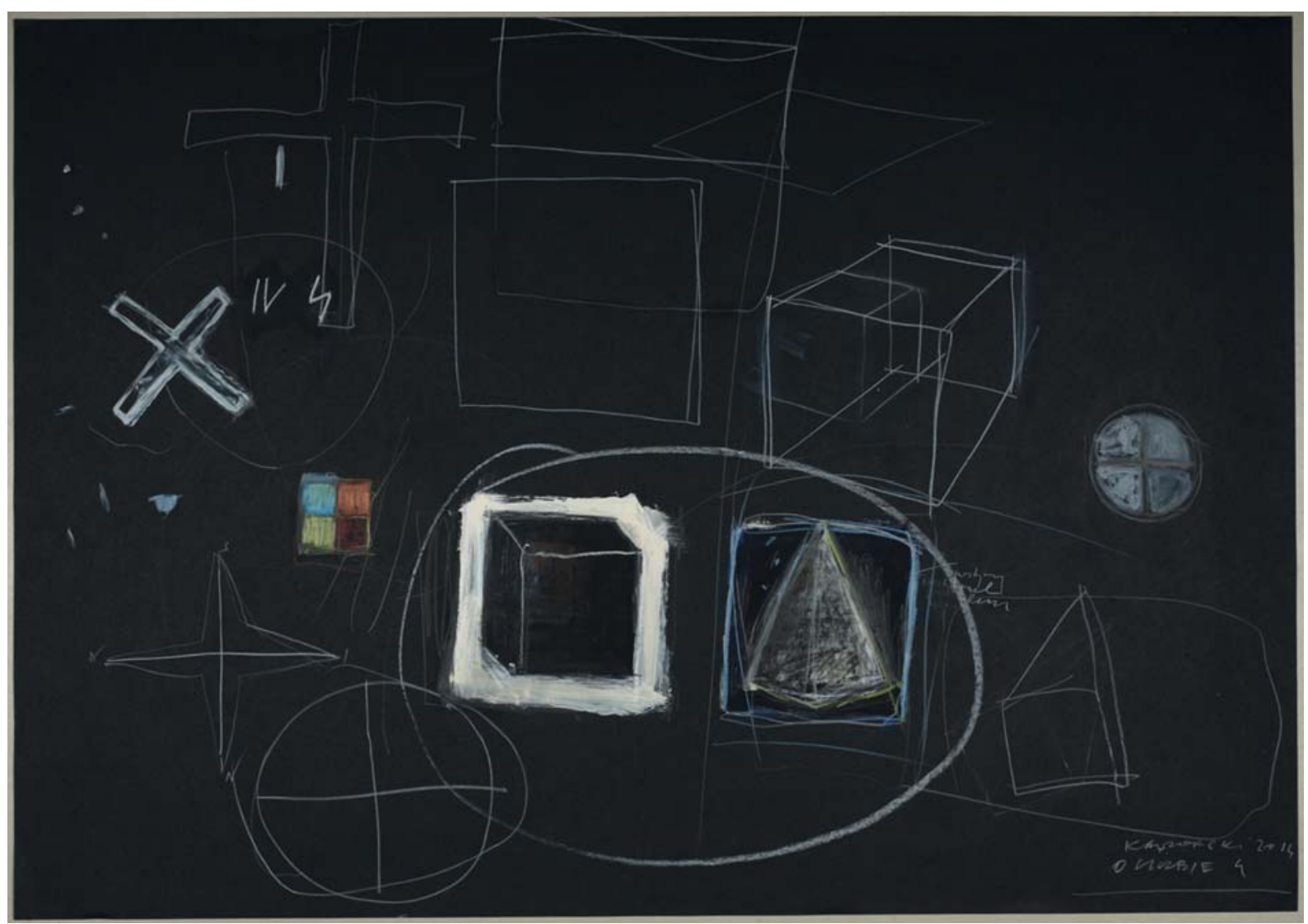

Jarosław Kawiorski, Über die Zahl 4, $70 \times 100 \mathrm{~cm}, 2014$, Wasserfarbe, Kreidestift 


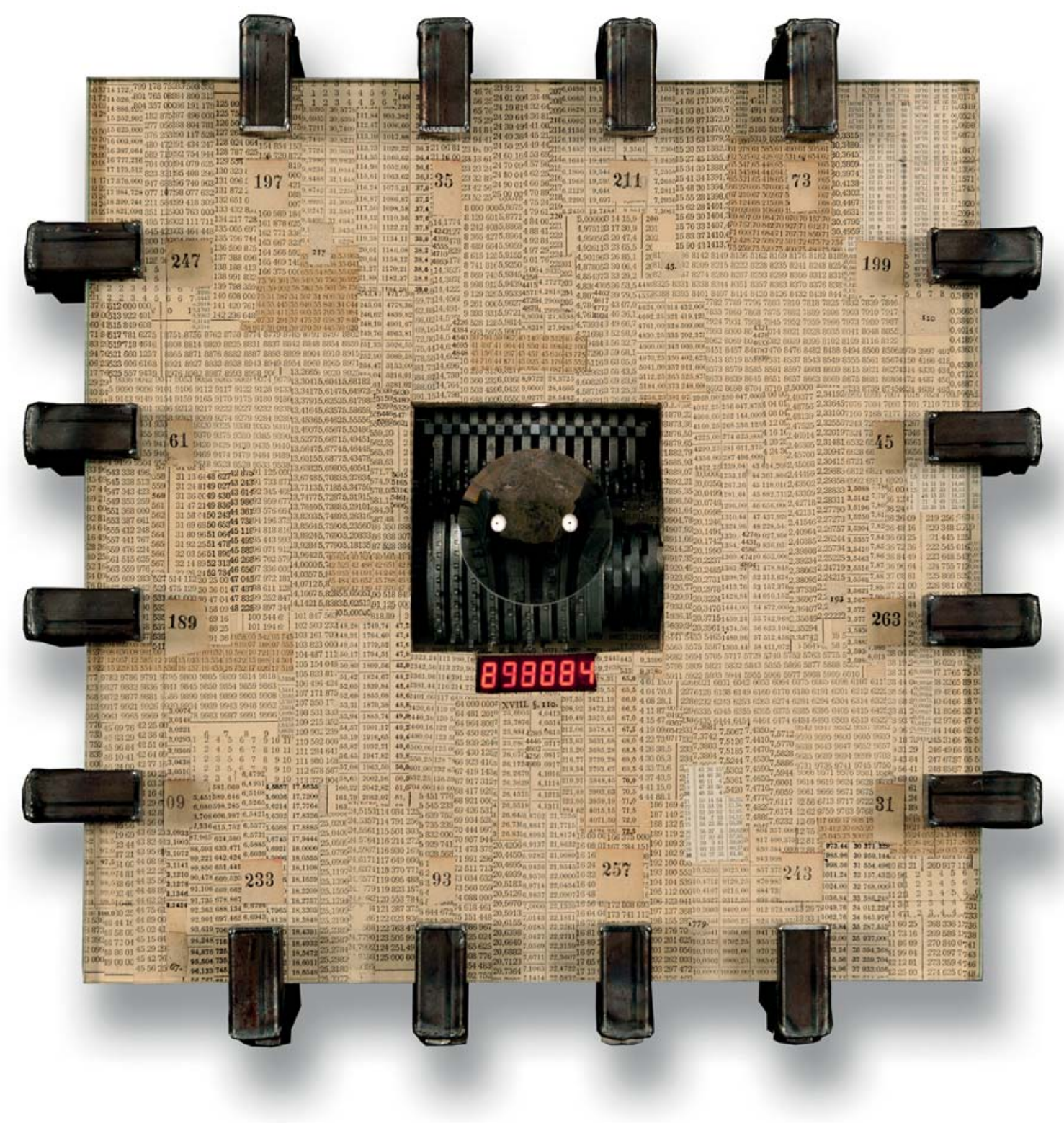

Andrzej Bednarczyk, Die Rattenfalle des Minotaurus, Algebra Welt, $39 \times 3 \times 13 \mathrm{~cm}, 2014$ 


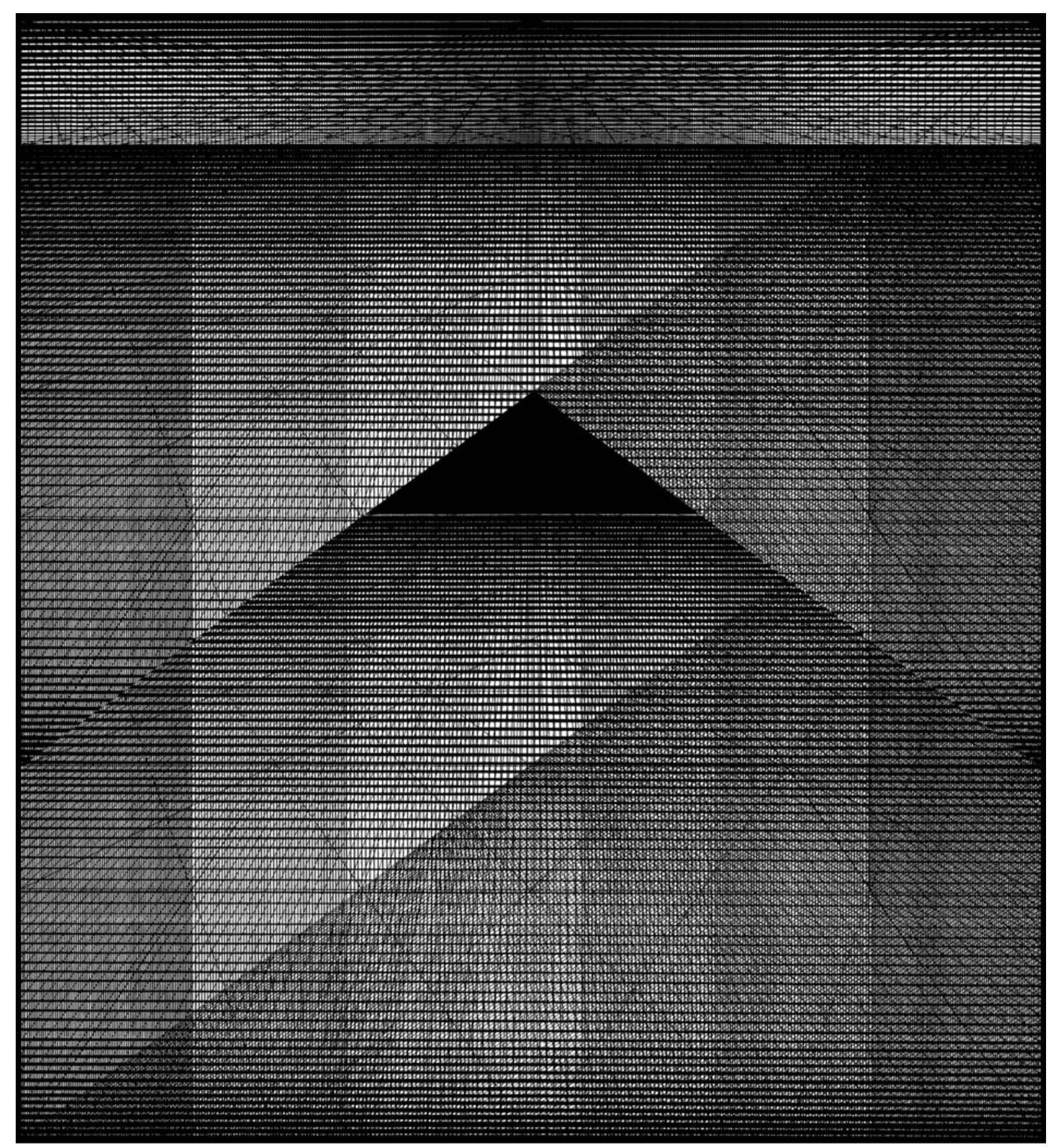

Tadeusz Wiktor, Erste Messe, Mottete 42, 74×67,5 cm, 198I, Offset, Collage 


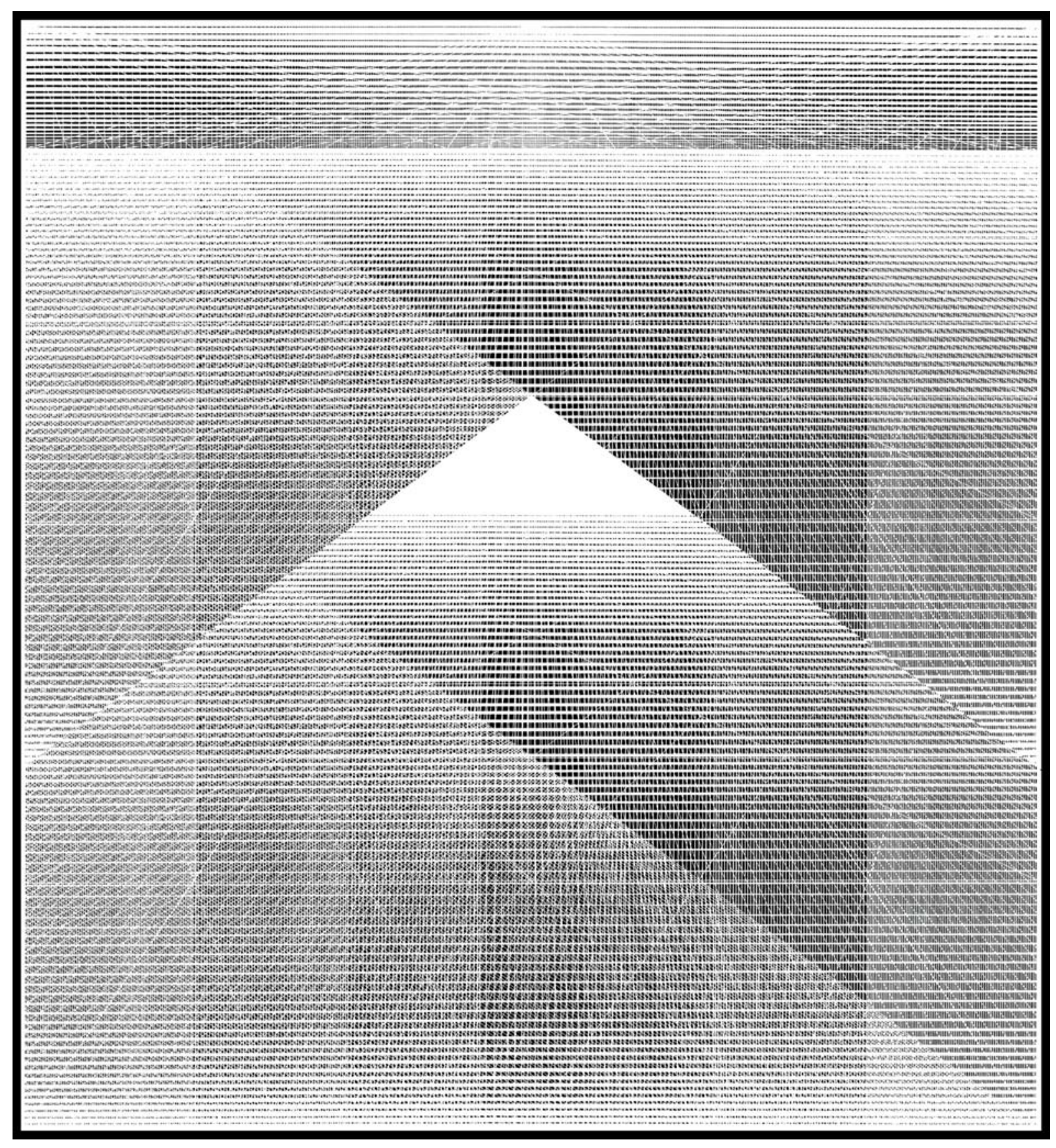

Tadeusz Wiktor, Erste Messe, Mottete 46, $74 \times 67,5 \mathrm{~cm}, 198$ I, Offset, Collage 


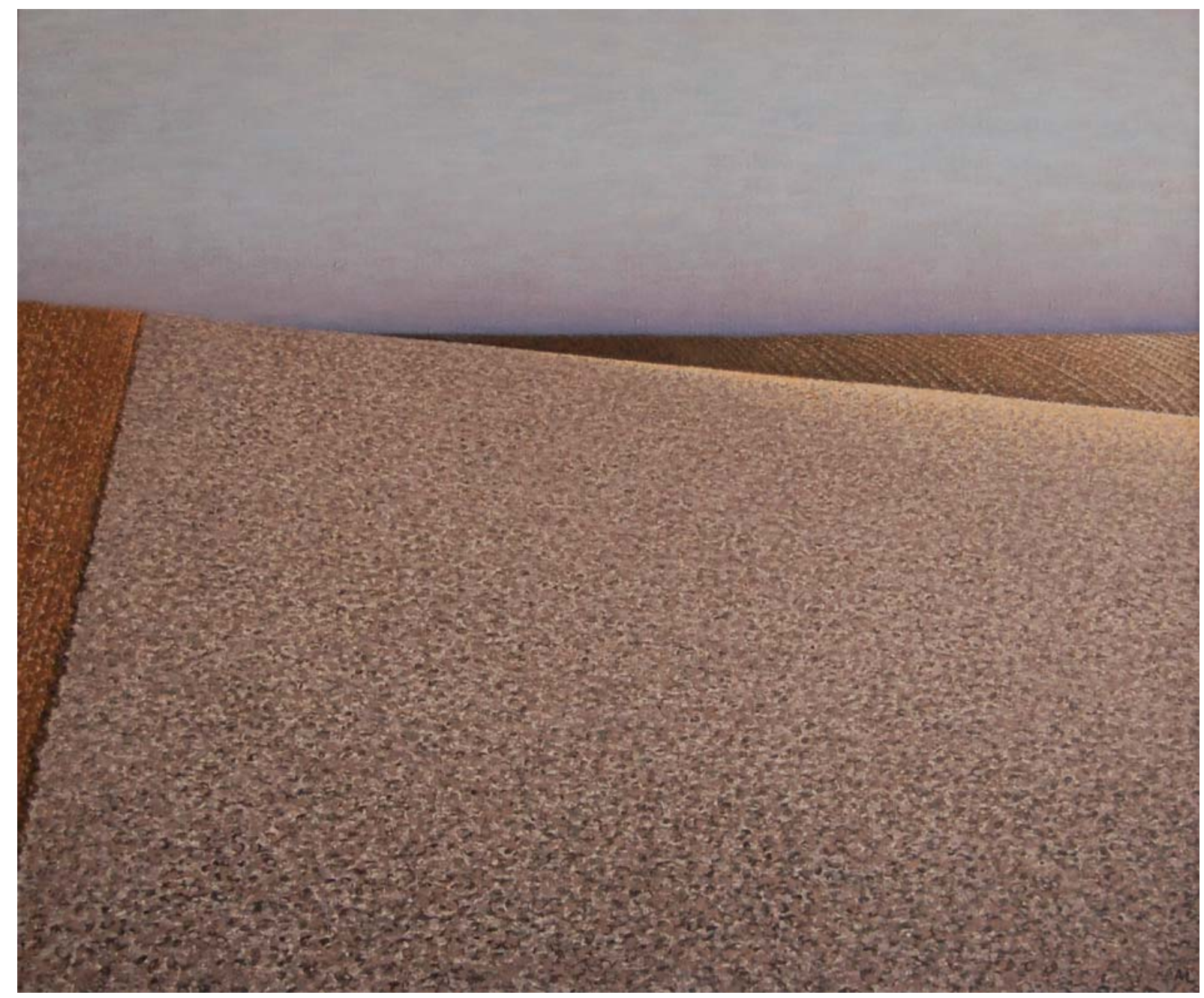

Leszek Misiak, Himmel und Erde, $100 \times 120 \mathrm{~cm}, 2008$, Tempera auf Leinwand 


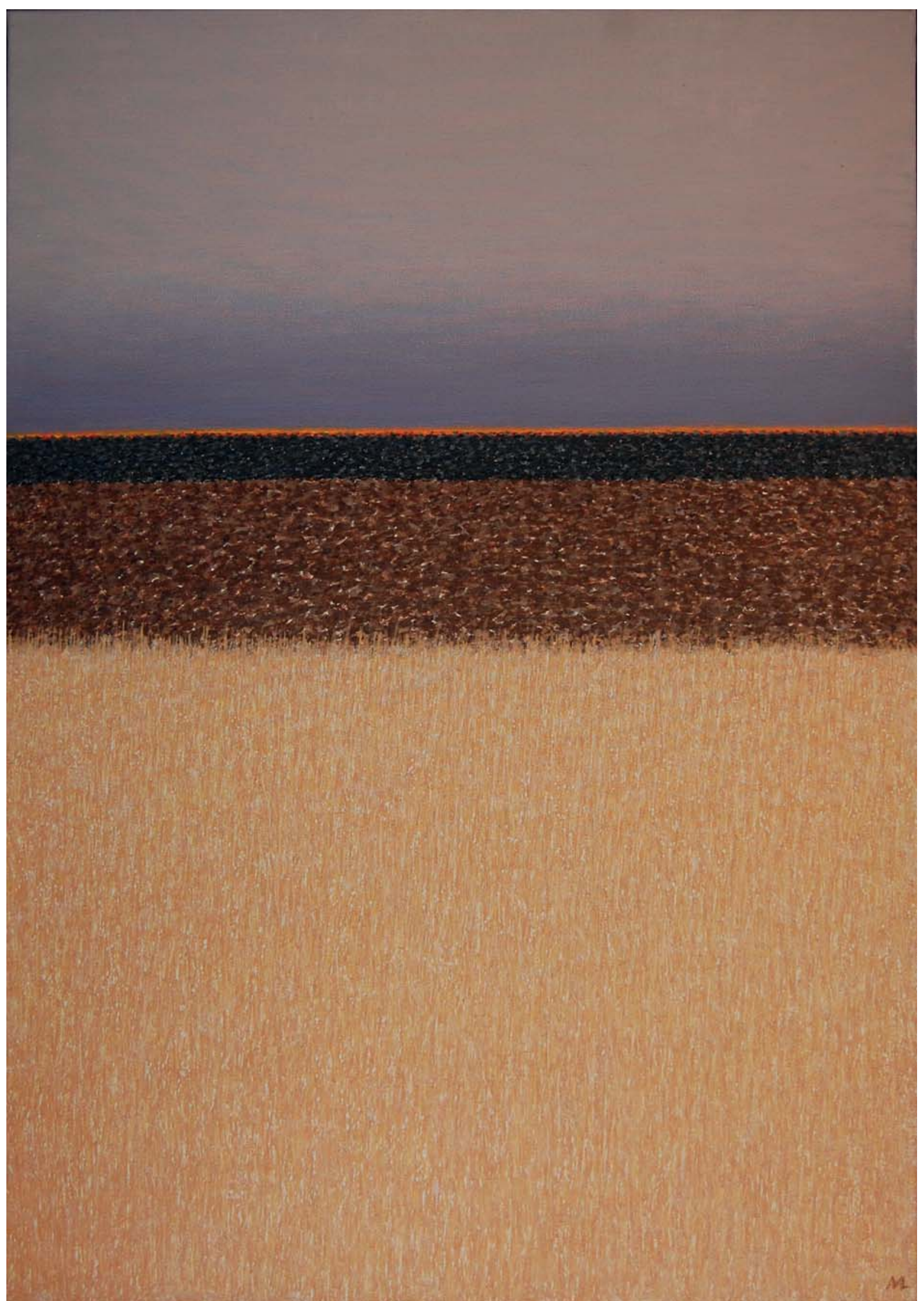

Leszek Misiak, Himmel und Erde, $100 \times 70 \mathrm{~cm}, 2010$, Tempera auf Leinwand 
Die Künstler

Andrzej Bednarczyk, geb. 1960 in Leśna. Studium an der Akademie der Bildenden Künste Krakau. Abschluss 1986. Professur 2009. Er arbeitet an der Akademie der Bildenden Künste Krakau an Gemälden, Grafiken, Fotografien sowie Zeichnungen und erstellt Installationen, Kunstobjekte und Buchkunst. Seine Arbeiten sind in den Sammlungen der Library of Congress, Washington, der British Library, London, der Nationalgalerie Prag, des Musashino Art University Museum \& Library, Tokyo, der Stanford University Library sowie in vielen anderen öffentlichen und privaten Sammlungen Polens und darüber hinaus zu finden.

Jarosław Kawiorski, geb. 1955 in Nowy Sạcz. Abschluss und Professur an der Akademie der Bildenden Künste Krakau. Er arbeitet an Malereien, Zeichnungen und Grafiken. Seine Arbeiten sind in den Sammlungen des Nationalmuseums in Krakau und vielen staatlichen Museen und Sammlungen Polens zu finden.

Leszek Misiak, geb. 1943. Abschluss an der Akademie der Bildenden Künste Krakau. Betreuung durch Prof. Wacław Taranczewski. Diplom mit Auszeichnung 1968. Professor an der Akademie der Bildenden Künste Krakau. Er arbeitet an Malereien, Zeichnungen und Grafiken und beteiligte sich an vielen Ausstellungen in Polen und im Ausland.
Seine Arbeiten sind in den Sammlungen des Nationalmuseums Krakau, dem Kunstmuseum Lodz, dem Musée d' Art et d' Histoire, Freiburg, dem Gewerbemuseum Winterthur, dem Museum of Contemporary Arts in Skopie, dem Portland Oregon Art Museum und in vielen Museen und staatlichen Sammlungen Polens zu finden.

Marian Warzecha, geb. 1930 in Krakau. Studium an der Jagellonische Universität und Akademie der Bildenden Künste Krakau. Mitglied der Krakau-Gruppe. Viele Einzelausstellungen in Polen und im Ausland. Seine Arbeiten werden auf vielen wichtigen Ausstellungen in Polen und im Ausland ausgestellt Seine Arbeiten sind in den Sammlungen aller wichtigen Museen Polens, dem Museum of Modern Art in New York sowie in vielen privaten Sammlungen Polens, der USA und anderer Ländern zu finden.

Tadeusz Gustaw Wiktor, geb. 1946 in Jelenia Góra. Studium der Malerei und Grafik an der Akademie der Bildenden Künste Krakau. Abschluss 1974. Professor an der Akademie der Bildenden Künste Krakau. Er arbeitet an Malereien, Zeichnungen und Grafiken, bereitete über 50 Einzelausstellungen vor und war an über 400 Ausstellungen in Polen und im Ausland beteiligt. 1988 erhielt er den Grand Prix, I I-ème Exposition Internationale de Dessins Originaux, Rijeka, und 2007 den Irene und Peter LudwigPreis, Print Internationale Grafik Triennale, Wien. Viele weitere Preise auf internationalen Malerei- und Zeichenausstellungen. 